\section{A nested case control study demonstrating increased chronic fatigue six years after a $Q$ fever outbreak}

\author{
Hugo C. van Woerden, ${ }^{1}$ Brendan Healy, \\ Meirion B. Llewelyn, ${ }^{3}$ Ian P. Matthews ${ }^{1}$ \\ 1Department of Primary Care \& Public \\ Health, Neuadd Meirionnydd; \\ 2Public Health Wales Microbiology \\ Cardiff, University Hospital of Wales, \\ Heath Park, Cardiff; \\ ${ }^{3}$ Royal Gwent Hospital, Cardiff Road, \\ Newport, UK
}

\section{Abstract}

The frequency and range of long-term sequelae following acute $Q$ fever infection remains controversial. This study aimed to assess a range of potential psychological and physical sequelae in a follow up study of a large outbreak of $Q$ fever that occurred in Newport, Wales, in 2002. A nested case control study was undertaken six years after a point source outbreak of $Q$ fever. We invited a cohort of 211 factory workers exposed to a point source of $Q$ fever in 2002 to attend a follow up clinic in 2008. Cases, defined as those who had clinical symptoms and serological evidence of acute $Q$ fever in 2002, were compared to controls, who worked in the same factory but were serologically negative at the time of the outbreak. At the follow up clinic blood was taken for Coxiella burnetii microimmunofluorescence and questionnaires were completed including the PHQ-9, Chalder Fatigue scale, and General Health Questionnaire. Results were obtained for 32 cases and 13 controls. Chalder Fatigue scores were significantly raised in the cases (independent samples t-test: $\mathrm{P}=0.047)$. $\mathrm{PHQ}-9$ and GHQ scores were not significantly raised in cases. However, post hoc cross sectional analysis indicated a relationship between Phase 2 IgG at follow up in 2008 and Chalder Fatigue scores $(\mathrm{P}=0.004)$ and $\mathrm{PHQ}-9$ scores (0.049). A longitudinal association was demonstrated between acute $Q$ fever infection and chronic fatigue six years later. In crosssectional analysis a previously unreported relationship between depression scores (PHQ9) and positive $Q$ fever serology was also identified.

\section{Introduction}

A number of follow up studies of patients with acute $Q$ fever ${ }^{1-8}$ and serological examina- tion of patients presenting with Chronic Fatigue Syndrome, ${ }^{9}$ have indicated that $Q$ fever may be associated with chronic fatigue. A relationship between previous $Q$ fever and symptoms of depression can also be postulated. ${ }^{10}$ The underlying aetiology of the association between $Q$ fever and chronic fatigue remains unclear. The extent to which chronic symptoms are influenced by the strain of $C$. burnetii, psychosocial factors, immunological or genetic factors is also uncertain. ${ }^{11,12}$ The possibility of long term effects following acute infection is supported by evidence that the aetiological organism can be detected in a proportion of patients by PCR many years later. ${ }^{13-15}$ It is still unclear whether patients that have long standing chronic fatigue after acute $Q$ fever gain any benefit from antibiotic treatment. ${ }^{16,17}$

A point source outbreak of $Q$ fever occurred in a cardboard manufacturing plant in Newport, South Wales, in 2002.18 This outbreak was one of the largest to have occurred in the UK. During the initial outbreak, the exposed cohort was identified and a nested case control study was undertaken to assess the difference in exposure between cases and controls to a range of potential sources of the outbreak. The cohort of factory workers, including affected and unaffected individuals provided the opportunity for a follow up study, which was conducted six years later in 2008 . The serological findings of the follow up study have been published. ${ }^{19}$ The current paper compares the symptoms of the original cases and controls in a follow up study. The aim of the study was to assess the hypotheses that i) chronic fatigue, ii) depression and iii) reduced physical function were more common in those who had acute $Q$ fever in 2002 compared to those individuals in the cohort who worked in the same factory but did not demonstrate evidence of infection at the time of the outbreak.

\section{Materials and Methods}

From the initial cohort of 226 individuals who worked in the factory in 2002 , all 131 serologically positive cases were invited to take part in the follow up study in 2008 , as were a random sample of 80 of the 95 serologically negative individuals. A written invitation to participate, including a leaflet outlining the proposed study, was therefore sent to 211 potential participants.

Those individuals who agreed to take part in the study were invited to a clinic where blood was taken for microimmunofluorescence testing for presence of Coxiella burnetii Phase 2 IgG and IgM antibodies and Phase 1 IgG and IgA antibodies. The microimmunofluorescence assays used antigen from Patient strain Lane -
Correspondence: Hugo C. van Woerden, Department of Primary Care \& Public Health, Neuadd Meirionnydd, Heath Park, Cardiff CF14 4YS, UK.

Tel. +44.07779.590991.

E-mail: vanwoerdenh1@cf.ac.uk

Key words: Q fever, Coxiella burnettii, chronic fatigue syndrome, chalder fatigue scale, PHQ-9

Received for publication: 11 June 2011. Accepted for publication: 9 September 2011.

This work is licensed under a Creative Commons Attribution NonCommercial 3.0 License (CC BYNC 3.0).

(C) Copyright H.C. van Woerden et al., 2011

Licensee PAGEPress, Italy

Microbiology Research 2011; 2:e19

doi:10.4081/mr.2011.e19

ST12 group. The blood samples were analysed by the standard reference laboratory in the UK, the Special Pathogens Reference Unit, HPA Centre for Emergency Preparedness and Response, Porton Down, Salisbury, SP4 0JG. Questionnaires including the PHQ- ${ }^{20}$ which assesses the severity of symptoms of depression, the 11 item version of the Chalder Fatigue scale, ${ }^{21}$ and the General Health Questionnaire (GHQ) which assesses physical health $^{22}$ were completed at the clinic. The questionnaire included two new single item tests for fatigue. Firstly, If you are tired at the moment how long has this lasted? Response options were not reported being tired, less than one week, one week to 3 months, 3 to 6 months, 6 months or more. For analysis the results were dichotomised into those who had fatigue for six months or more and those who had fatigue for less than six months. Secondly, the questionnaire asked, What percent of the time do you feel tired? Response options were $0 \%, 25 \%$, $50 \%, 75 \%$, or $100 \%$ of the time. Responses were dichotomised into those who reported being tired less than 50\% of the time and 50\% of the time or more.

The PHQ-9 is a self-administered subset of the PRIME-MD diagnostic instrument for common mental disorders. It is a widely used and well validated measure of depression based on nine questions that start with the stem, Over the last 2 weeks, how often have you been bothered by any of the following problems? Answers range from 0 (not at all) to 3 (nearly every day). The Chalder Fatigue scale used in this analysis contains 11 questions covering the physical and mental symptoms of fatigue including, tiredness, need to rest, feeling drowsy, problems starting things, and difficulty concentrating. The General Health Questionnaire is a broad ranging and widely used measure of physical health. The two other questions 
on fatigue duration and percent of time affected by fatigue were not previously validated questions.

Questionnaire data was analysed using Microsoft Excel ${ }^{\circledR}$ and SPSS ${ }^{\circledR}$. In view of the relatively small sample sizes, the non-parametric Mann-Whitney $U$ test for two independent samples was used to compare variables. Variables were also dichotomised and compared using the chi-square test. Fishers' exact test was used where where one cell had an expected count of less than five.

Ethical approval for the study was granted by the South East Wales Research Ethics Committee Panel C.

\section{Longitudinal and cross sectional analyses}

The data was analysed as a nested case control study, based on the status of participants in 2002 as cases or controls. In 2002 cases were defined as those members of the exposed cohort who had clinical symptoms of acute $Q$ fever infection and serological evidence of infection as demonstrated by a Phase 2 IgM $\geq 80$, or a fourfold rise on sequential Complement Fixation Tests (CFT). Non-cases were defined as those who had no symptoms of acute $Q$ fever infection and no serological evidence of infection with no IgM, no CFT and no Phase 1 or 2 IgG. Three patients who did not unambiguously meet criteria as cases or controls were excluded from analysis. They had uncertain serology defined as no IgM, no fourfold rise in CFT but CFT $\geq 8$ or Phase 1 or 2 IgG $>160$. Two of the three individuals with uncertain serology had symptoms; the third individual did not. These three individuals were excluded from all the analysis presented in this paper.

A post hoc cross sectional analysis of the cohort was also undertaken, assessing the relationship between current clinical symptoms at the time of the clinic in 2008 and Phase 2 IgG blood results from samples taken at the same time. Phase $2 \operatorname{IgG}$ titres were recorded as $0,320,640,1280$, or 2560 . Scores for clinical symptoms were dichotomised as follows: Chalder Fatigue (0-3, 4-11); PHQ-9 scores (dichotomised at the median: 0-3, 4-9); duration of tiredness (less than 6 months, 6 months or more); percent of time participants felt tired (less than 50\% of the time, $50 \%$ of the time of more); and GHQ scores (dichotomised at the median: $0-2,3-12$ ).

\section{Results}

Results for analysis were available for 45 individuals out of a total of 52 individuals who attended the follow up clinic. This represented
$24.4 \%$ of cases invited to the clinic (32/131) and $16.3 \%$ of the controls invited to the follow up clinic (13/80). The characteristics of the cohort used for the nested case control study are shown in Table 1. Both groups were predominantly male, reflecting the population in the factory where the outbreak occurred. The mean age of cases was 50.18 yrs (SD 9.85, range 27-64 yrs) and the mean age of controls was $53.57 \mathrm{yrs}$ (SD 8.86, range 36-68 yrs). Educational attainment was similar in both groups: $37.5 \%$ (12/32) of cases and $38.5 \%$ $(5 / 13)$ of controls had remained in education after the minimum school leaving age.

\section{Nested case control study}

A table showing the relationship between status in 2002 and symptoms in 2008 is provided in Table 2. Raised Chalder Fatigue scores were more common in cases $(\mathrm{P}=0.047)$. The single questions analysing symptoms of tiredness for 6 months or more or tiredness for 50\% of the time or more were not statistically significantly associated with being a case. Neither were $\mathrm{PHQ}-9$ scores $(\mathrm{P}=0.189)$ or $\mathrm{GHQ}$ scores $(\mathrm{P}=0.743)$.

\section{Cross sectional analysis}

A second post hoc analysis of the cohort was

Table 1. Summary of cases and controls (bases on status in 2002) by gender.

\begin{tabular}{lccc} 
& Case & Status & Total \\
Gender & & Control & \\
Female & 7 & 1 & 8 \\
Male & 28 & 13 & 44 \\
Total & 35 & 14 & 52 \\
\hline
\end{tabular}

Table 2. Relationship between status as defined in 2002 (cases and controls) and clinical symptoms in 2008.

\begin{tabular}{|c|c|c|c|c|c|c|}
\hline Comparison & $\mathbf{N}$ & & & & cols & $\begin{array}{c}\text { Fisher's } \\
\text { Exact } \\
\text { test P }\end{array}$ \\
\hline Chalder fatigue score & & $0-3$ & $4-11$ & $0-3$ & $4-11$ & \\
\hline & 45 & 13 & 19 & 10 & 3 & 0.047 \\
\hline Tired for 6 months or more & & 6 mont & 26 month & 6 mont & $\geq 6$ months & \\
\hline$\gamma$ & 39 & 11 & 17 & 8 & 3 & 0.082 \\
\hline Tired for $50 \%$ of the time or more & & $0-50 \%$ & $50-100 \%$ & $0-50 \%$ & $50-100 \%$ & \\
\hline r & 40 & 7 & 21 & 6 & 6 & 0.154 \\
\hline PHQ-9 & & $1-3$ & $4-9$ & $1-3$ & $4-9$ & \\
\hline 1$)$ & 45 & 14 & 18 & 9 & 4 & 0.189 \\
\hline GHQ & & $0-2$ & $3-12$ & $0-2$ & $3-12$ & \\
\hline & 49 & 22 & 13 & 10 & 4 & 0.743 \\
\hline
\end{tabular}

Table 3. Relationship between Phase 2 IgG in 2008 and symptoms measured at the same time.

\begin{tabular}{llcccc} 
& Comparison & N & Mean rank & $\begin{array}{c}\text { Sum of } \\
\text { Ranks }\end{array}$ & $\begin{array}{c}\text { Mann- } \\
\text { whitney U }\end{array}$ \\
Phase 2 IgG & & & 412.00 & 136.000 \\
& Chalder Fatigue score (0-3) & 23 & 17.91 & 623.00 & $\mathrm{P}=0.004$ \\
& Chalder Fatigue score (4-11) & 22 & 28.32 & & \\
& Total & 45 & & & \\
Phase 2 IgG & Tired for 6 months or more & 19 & 16.45 & 312.50 & 122.500 \\
& Not tired for 6 months or more & 20 & 23.38 & 467.50 & $\mathrm{P}=0.037$ \\
& Total & 39 & & & \\
\hline Phase 2 IgG & Tired less than 50\% of the time & 13 & 14.69 & 191.00 & 100.000 \\
& Tired 50\% of the time or more & 27 & 23.30 & 629.00 & $\mathrm{P}=0.017$ \\
& Total & 40 & & & \\
Phase 2 IgG & PHQ-9 score (0-3) & 23 & 19.52 & 449.00 & 173.000 \\
& PHP-9 score (4-9) & 22 & 26.64 & 586.00 & $\mathrm{P}=0.049$ \\
& Total & 45 & & & \\
\hline Phase 2 IgG & GHQ score (0-2) & 32 & 23.86 & 763.50 & 235.500 \\
& GHQ score (3-12) & 17 & 27.15 & 461.50 & $\mathrm{P}=0.406$ \\
& & 49 & & & \\
& & & & &
\end{tabular}


undertaken to assess whether symptoms recorded at the follow up clinic in 2008 were associated with Phase 2 IgG results taken at the same time (Table 3). There was a clear relationship between higher $C$. burnetii Phase $2 \mathrm{IgG}$ and raised Chalder Fatigue score $(\mathrm{P}=$ $0.004)$, tiredness for 6 months or more $(\mathrm{P}=$ $0.037)$, tiredness for $50 \%$ of the time or more $(\mathrm{P}=0.012)$, and raised PHQ-9 score (0.049), but not GHQ score $(\mathrm{P}=0.406)$.

Similarly, dichotomising $C$. burnetii Phase 2 IgG scores to $0-640$ and $1280-2560$ and assessing the relationship with symptoms using the chi-square test indicated a clear relationship to clinical symptoms of fatigue: Chalder Fatigue score (chi-square 8.049; $\mathrm{P}=0.005$ ), tiredness for 6 months or more (chi-square 4.311; $\mathrm{P}=0.038$ ), tiredness for $50 \%$ of the time or more (chi-square 6.686; $\mathrm{P}=0.01$ ). Relationships with PHQ-9 score (chi-square 2.702; $\mathrm{P}=0.1$ ), and GHQ score (chi-square $0.634 ; \mathrm{P}=0.426$ ) were not statistically significant. Four of the fourteen control subjects (based on their status in 2002) had $C$. burnetii Phase 2 IgG titres of 320 for $Q$ fever at follow up in 2008.

\section{Discussion}

Chalder Fatigue scores were significantly raised six years later in those individuals who were diagnosed with acute $Q$ fever at the time of the outbreak. In contrast, general health, as measured by the GHQ, was not significantly different from controls drawn from the same cohort of individuals who worked in the same factory at the time of the outbreak. There is a statistically significant cross-sectional relationship $(\mathrm{P}=0.049)$ at the time of follow up between raised serology by microimmunofluorescence and both Chalder Fatigue scores and depression as measured by the PHQ-9. Symptoms of fatigue and depression can overlap and can be difficult to differentiate. However, as these results were derived from the cohort of workers exposed to $Q$ fever in the initial outbreak in 2002, there is some evidence to suggest that depression may be a associated with previous $Q$ fever infection. This finding will need verified by future studies. An unexpectedly large proportion of the controls (28.6\%) were serologically positive at follow up. This figure is much higher than the sero-prevalence of $Q$ fever in the UK population. ${ }^{23}$ The positive $Q$ fever serology may represent late sero-conversion due to exposure at the time of the outbreak, later exposure to $Q$ fever, or false positive results.

The main strength of this study was that it used validated questionnaires to assess symptoms associated with the long term sequelae of $\mathrm{Q}$ fever subsequent to a point source outbreak.
The study was also strengthened by the fact that controls were drawn from the same environment as cases. However, some controls may indeed have been unidentified asymptomatic cases and a separate population of control subjects would have strengthened this study further. The potential inclusion of some cases in the control group would reduce the power of the study to detect an effect and should, therefore, not have introduced bias in favour of our findings.

The sample size in this study is small and the response rate is relatively low. Participants were aware whether or not they had been diagnosed as having acute $Q$ fever in initial outbreak and this may have introduced some reporting bias when completing the follow up questionnaires. Potential problems in interpreting serological results and defining international standards for acute, past and chronic infection have been considered in a previous report. ${ }^{19}$

It would have been helpful to have information on other blood borne infections that could have contributed to chronic fatigue. However, we have no reason to suspect that the prevalence of such infections would be different in cases and controls. Consequently, this issue should not have materially affected our findings. It would have been interesting to assess whether there was a relationship between the nature of the work undertaken by each participant in the study and their level of fatigue. In particular, whether there was a relationship between the symptoms of chronic fatigue and the level of physical exertion undertaken on a day to day basis or the shift pattern worked by the individuals concerned. It would also have been useful to compare the frequency and duration of sick leave in cases and controls. Unfortunately, none of this information was collected. One patient from this cohort developed $Q$ fever endocarditis and was treated with antibiotics. However, it is unclear whether or not chronic fatigue after $Q$ fever infection should be treated with antibiotics. Raised Q fever serology is more common in patients with Chronic Fatigue Syndrome than in the background population. ${ }^{9}$ Small studies have shown that some patients with chronic fatigue and raised $Q$ fever serology respond to antibiotic treatment. ${ }^{16,24}$ However, high antibody levels may indicate high responder status rather than the actual presence of the microorganism. This study points up the desirability of a trial of antibiotics in patients with post $\mathrm{q}$ fever chronic fatigue.

\section{Conclusions}

This study has demonstrated that chronic fatigue was more common six years later in patients who were infected with $Q$ fever during an outbreak. The study has also raised the possibility of a relationship between raised $C$. burnetii Phase 2 IgG and symptoms of chronic fatigue and depression.

\section{References}

1. Hatchette TF, Hayes M, Merry H, et al. The effect of $\mathrm{C}$. burnetii infection on the quality of life of patients following an outbreak of Q fever. Epidemiol Infect 2003;130:4915.

2. Ayres JG, Flint N, Smith EG, et al. Postinfection fatigue syndrome following $Q$ fever. QJM 1998;91:105-23.

3. Ayres JG, Smith EG, Flint N. Protracted fatigue and debility after acute $\mathrm{Q}$ fever. Lancet 1996;347:978-9.

4. Ayres JG, Wildman M, Groves J, et al. Longterm follow-up of patients from the $1989 \mathrm{Q}$ fever outbreak: no evidence of excess cardiac disease in those with fatigue. QJM 2002;95:539-46.

5. Wildman MJ, Smith EG, Groves J, et al. Chronic fatigue following infection by Coxiella burnetii ( $Q$ fever): ten-year follow-up of the 1989 UK outbreak cohort. QJM 2002;95:527-38.

6. Limonard GJM, Peters JB, NabuursFranssen $\mathrm{MH}$, et al. Detailed analysis of health status of $Q$ fever patients 1 year after the first Dutch outbreak: a case-control study. QJM 2010;103:953-8.

7. Delsing CE, Kullberg BJ, Q fever in the Netherlands: a concise overview and implications of the largest ongoing outbreak. Netherlands J Med 2008;66:365-7.

8. Gale M, Ketheesan N, Govan B, et al. Q fever cases at a North Queensland centre during 1994-2006. Intern Med J 2007;37: 644-6.

9. Devanur LD, Kerr JR. Chronic fatigue syndrome. J Clin Virol 2006;37:139-50.

10. Morroy G, Peters J, van Nieuwenhof M, et al. The health status of $Q$-fever patients after long-term follow-up. BMC Infect Dis 11:97.

11. Helbig KJ, Heatley SL, Harris RJ, et al. Variation in immune response genes and chronic Q fever. Concepts: preliminary test with post- $Q$ fever fatigue syndrome. Genes Immun 2003;4:82-5.

12. Penttila IA, Harris RJ, Storm P, et al. Cytokine dysregulation in the post-Q-fever fatigue syndrome. QJM 1998;91:549-60.

13. Harris RJ, Storm PA, Lloyd A, et al. Longterm persistence of Coxiella burnetii in the host after primary Q fever. Epidemiol Infect 2000;124:543-9.

14. Kato K, Arashima Y, Asai S, et al. Detection of Coxiella burnetii specific DNA in blood 
samples from Japanese patients with chronic nonspecific symptoms by nested polymerase chain reaction. FEMS Immunol Med Microbiol 1998;21:139-44.

15. Marmion BP, Storm PA, Ayres JG, et al. Long-term persistence of Coxiella burnetii after acute primary Q fever. QJM 2005;98: 7-20.

16. Iwakami E, Arashima Y, Kato K, et al. Treatment of chronic fatigue syndrome with antibiotics: pilot study assessing the involvement of Coxiella burnetii infection Intern Med 2005;44:1258-63.

17. Ledina D, Bradaric N, Milas I, et al. Chronic fatigue syndrome after $Q$ fever.
Med Sci Monit 2007;13:CS88-92.

18. van Woerden HC, Mason BW, Nehaul LK, et al. Q fever outbreak in industrial setting. Emer Infect Dis 2004;10:1282-9.

19. Healy B. Chronic Q Fever: Different Serological Results in Three CountriesResults of a Follow-up Study 6 Years After a Point Source Outbreak. Clin Infect Dis 2011;52:1013-9.

20. Kroenke K, Spitzer RL, Williams JBW. The PHQ 9. J Gen Intern Med 2001;16:606-13.

21. Chalder T, Berelowitz G, Pawlikowska T, et al. Development of a fatigue scale. J Psychosom Res 1993;37:147-53.

22. Goldberg DP, Hillier VF. A scaled version of the General Health Questionnaire. Psychol Med 1979;9:139-45.

23. Thomas DR, Treweek L, Salmon RL, et al. The risk of acquiring $Q$ fever on farms: a seroepidemiological study. Occup Environ Med 1995;52:644-7.

24. Arashima Y, Kato K, Komiya T, et al. Improvement of chronic nonspecific symptoms by long-term minocycline treatment in Japanese patients with Coxiella burnetii infection considered to have post- $Q$ fever fatigue syndrome. Intern Med 2004; 43:49-54. 